\title{
Periprosthetic Infection on a Revised Total Hip Prosthesis Treated with Deep Lavage and Vacuum Dressings: A Case Report with 4-Year Follow-Up
}

\author{
Mohamad Darwish Hicham Abdel Nour Elias Saidy Dany Aouad \\ Georgio Lati Jason Hanna Alexandre Nehme \\ Department of Orthopedic Surgery and Traumatology, Saint George Hospital University \\ Medical Center, University of Balamand, Beirut, Lebanon
}

\section{Keywords}

Periprosthetic hip infection · Vacuum dressing · Debridement · Salvage

\begin{abstract}
Periprosthetic hip infections are a dreaded complication met among most orthopedic surgeons after arthroplasty procedures especially in susceptible patients. We report a case of a patient with periprosthetic infection after a revised total hip arthroplasty. She was treated with deep lavage and debridement, combined with IV anti-biotherapy and vacuum dressings. A 4-year follow-up shows an infection-free patient with an acceptable functional status and quality of life. Suppression of the infection, with salvage of the prosthesis and maintaining an acceptable functional status, is a satisfactory result that can be achieved in selected cases.
\end{abstract}

\section{Introduction}

Periprosthetic infections are commonly met among most orthopedic surgeons after arthroplasty procedures. They prevail in 1-2\% of primary joint arthroplasties, and up to $4 \%$ of revisions [1]. The infecting organisms form a biofilm which limits the effectiveness of antibiotherapy and thus making salvage of the infected prosthesis a challenging mission. This may necessitate various major surgeries including resection arthroplasties, arthrodesis, 1- or 2 -staged revision, and possible eventual amputation [2]. Periprosthetic infections are categorized as either acute or chronic. The duration between surgery and the onset of symptoms, in addition to the duration of symptoms, help in evaluating the maturation stage of the current 


\section{Case Reports in Orthopedic Research}
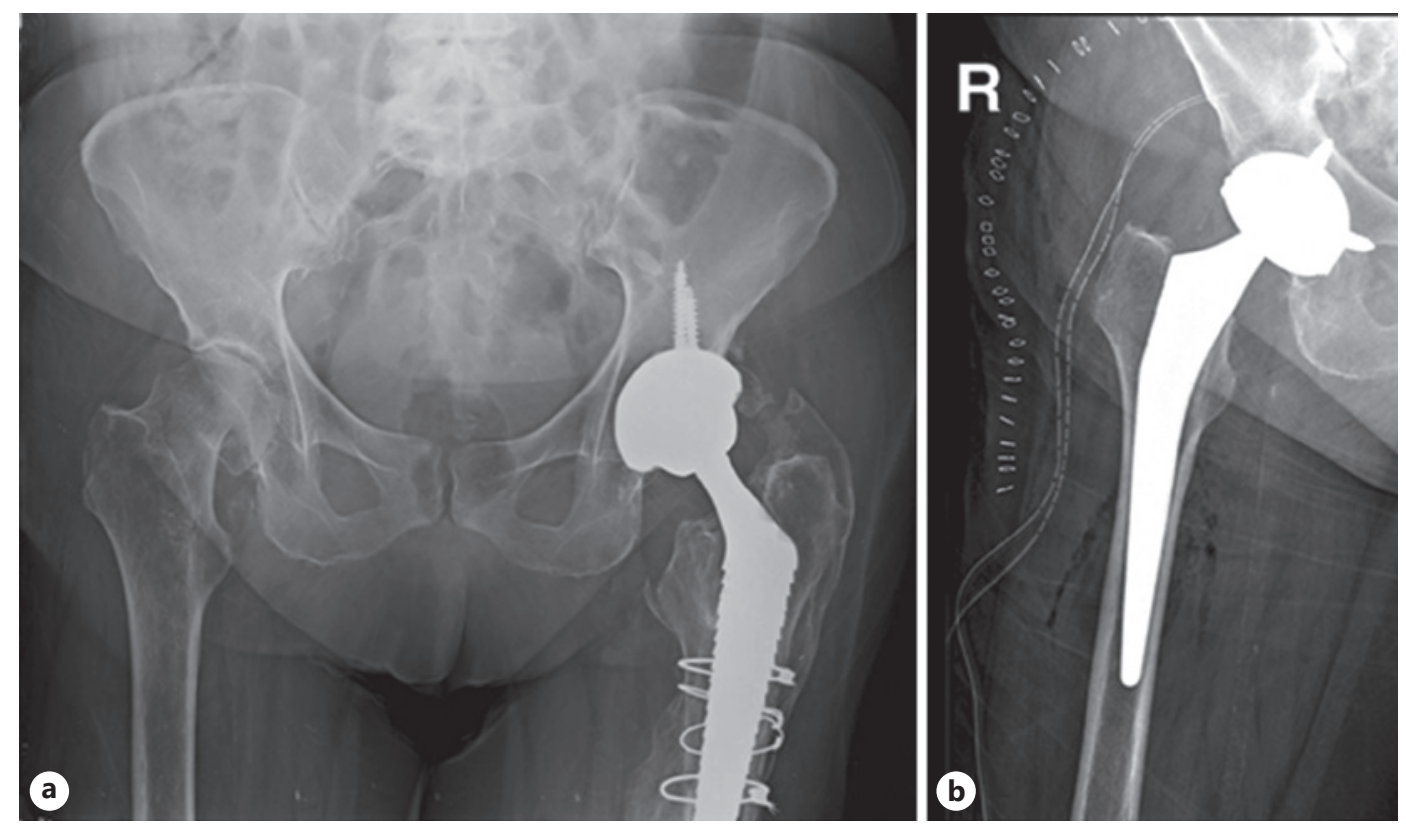

Fig. 1. AP pelvis X-ray showing a right displaced femoral neck fracture (a), Post-op right hip AP view after treatment with right total hip replacement (b).

biofilm which aids in the decision whether salvage or prosthesis exchange is to be decided [1]. Conservative treatment can be the only choice in selected cases where surgical intervention is not tolerated due to comorbidities or refusal by the patient [3]. Treatment with salvage of the prosthesis is reported by many authors; however, the results are highly variable in context of absence of uniform treatment protocols. In this article, we present a case of a patient with periprosthetic infection after a revised total hip arthroplasty. She was treated with deep lavage and debridement, combined with IV anti-biotherapy and vacuum dressings. A 4-year follow-up shows an infection-free patient with an acceptable functional status and quality of life.

\section{Case Presentation}

This is the case of a 89-year-old female who is known to have hypertension, diabetes mellitus type 2, dyslipidemia, and coronary artery disease. She has been operated 1 month prior to her presentation for a right femoral neck fracture (Fig. 1a) treated by a primary hip prosthesis (Fig. 1b). Her postoperative course was uneventful, and she was discharged home in stable condition with a full weight-bearing physical therapy protocol.

After this hospitalization, the patient was admitted into an elderly home, 2 weeks after which the patient sustained a fall in the elderly home and was brought to the emergency department where she was found to have a right femoral periprosthetic fracture with loose stem (Fig. 2). Revision of the femoral component is done replacing the stem with an anatomic modular revision stem with diaphyseal support, combined with a trochanteric plate fixed with cerclage wires (Fig. 3). During the operation, cultures were taken and extensive lavage was performed with proper debridement. The wound cultures as well as additional urine cultures were negative. She was given $2 \mathrm{~g}$ of cefazolin $30 \mathrm{~min}$ prior to incision. Her postoperative course was uneventful, and she was discharged home with a partial weight-bearing protocol with regularly monitored dressing changes done twice weekly.

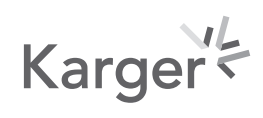




\section{Case Reports in Orthopedic Research}
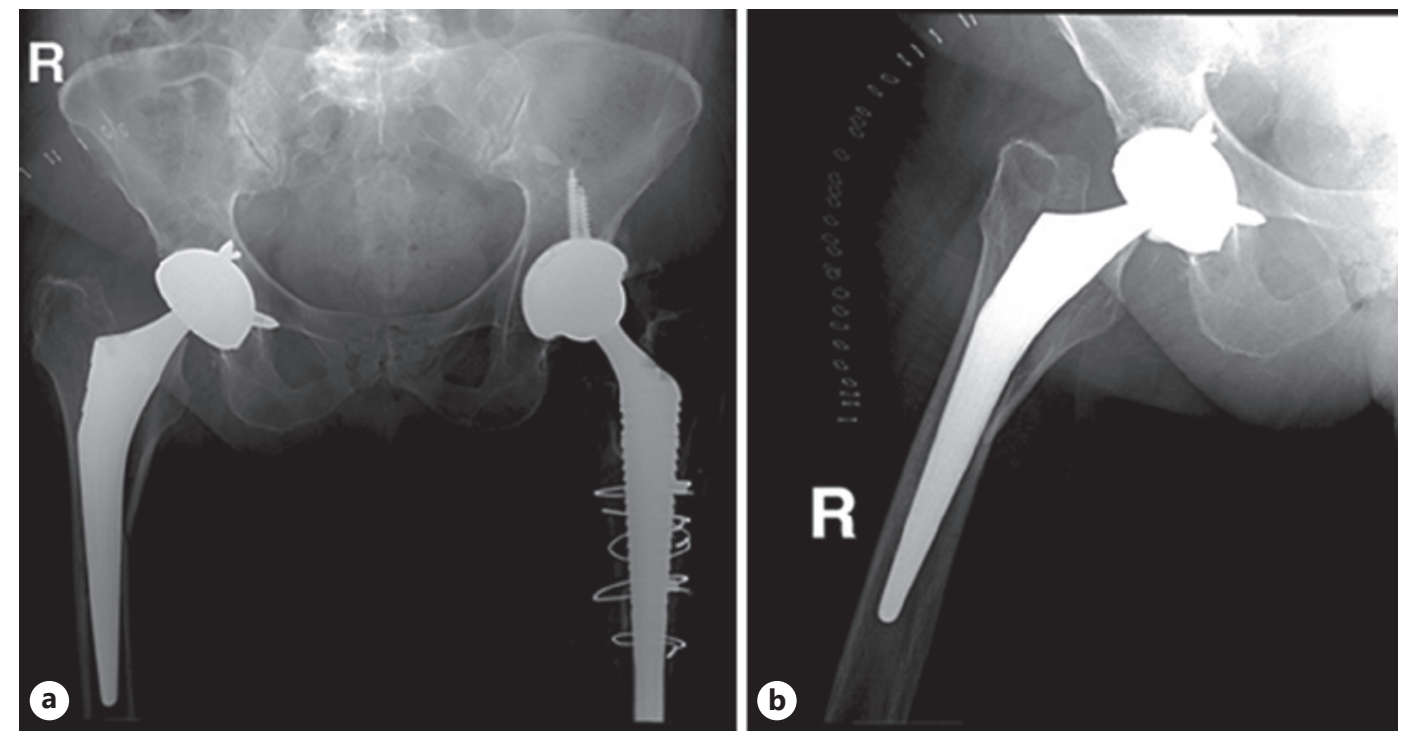

Fig. 2. AP pelvis (a) and lateral (b) views X-ray of the right hip showing a periprosthetic fracture with a loose stem.

Fig. 3. AP view postoperative X-ray of the right hip joint after revision of the femoral component for periprosthetic fracture.

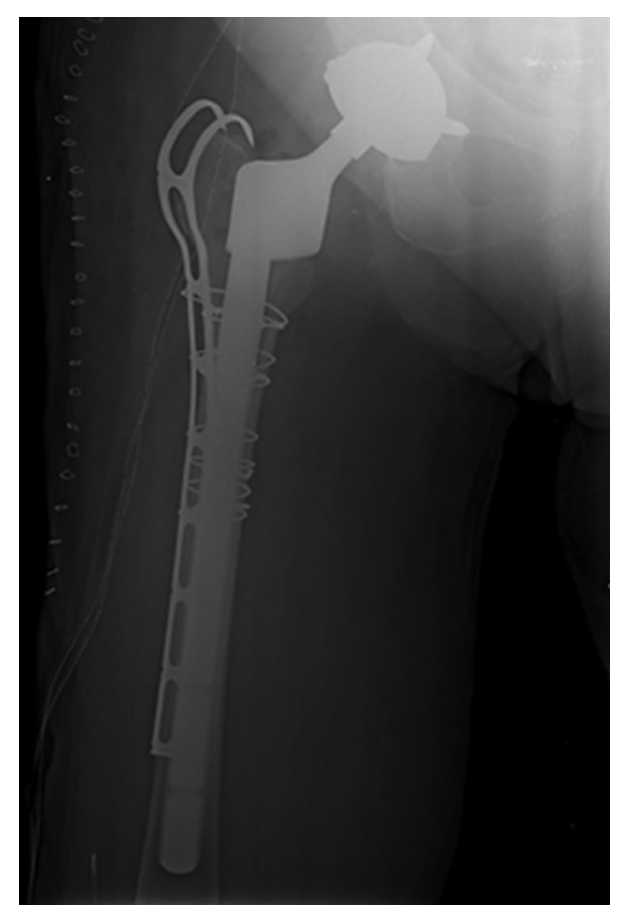

One month postoperatively, the patient presented back to the hospital after her surgical wound was found to be oozing pus during her routine wound care visit. The wound was erythematous and warm with visible pus oozing. Infection is diagnosed after laboratory workup showing elevated inflammatory markers, and a CT-scan showing diffuse air infiltration surrounding the prosthesis (Fig. 4). The patient was scheduled for operative lavage and debridement. Deep debridement is done with removal of the trochanteric plate (Fig. 5), and extensive lavage with $12 \mathrm{~L}$ of normal saline was done, and cultures were taken from the periprosthetic space followed by closure and placement of 2 vacuum drains. 


\section{Case Reports in Orthopedic Research}

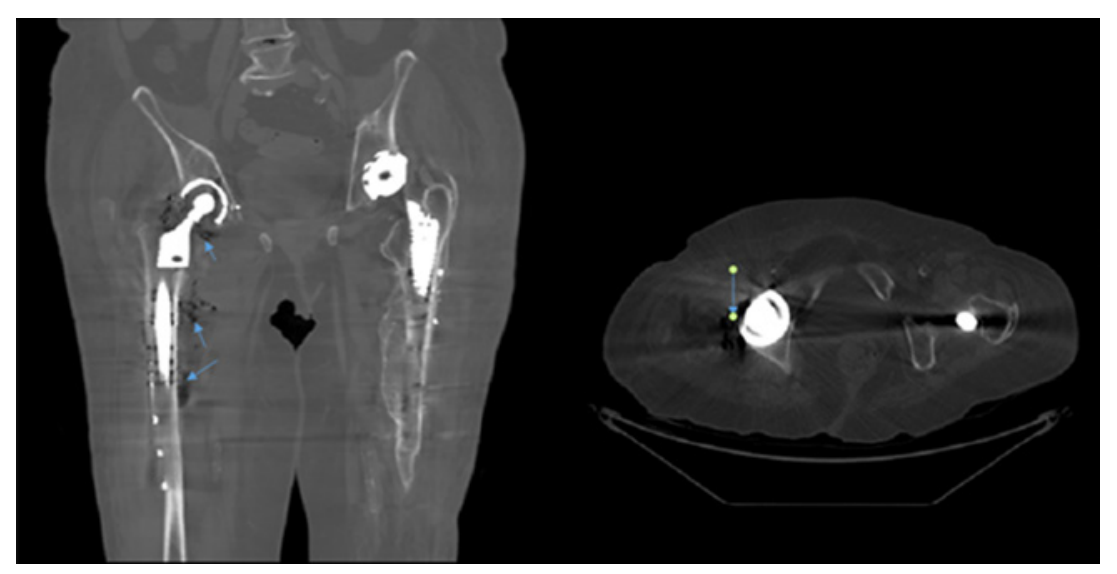

Fig. 4. Coronal and axial cuts of the pelvis and hip CT-scan showing diffuse air bubbles (arrows) surrounding the right total hip prosthesis in context of infected wound and elevated inflammatory markers.

Fig. 5. AP view X-ray of the right hip after removal of the trochanteric plate and cerclages.

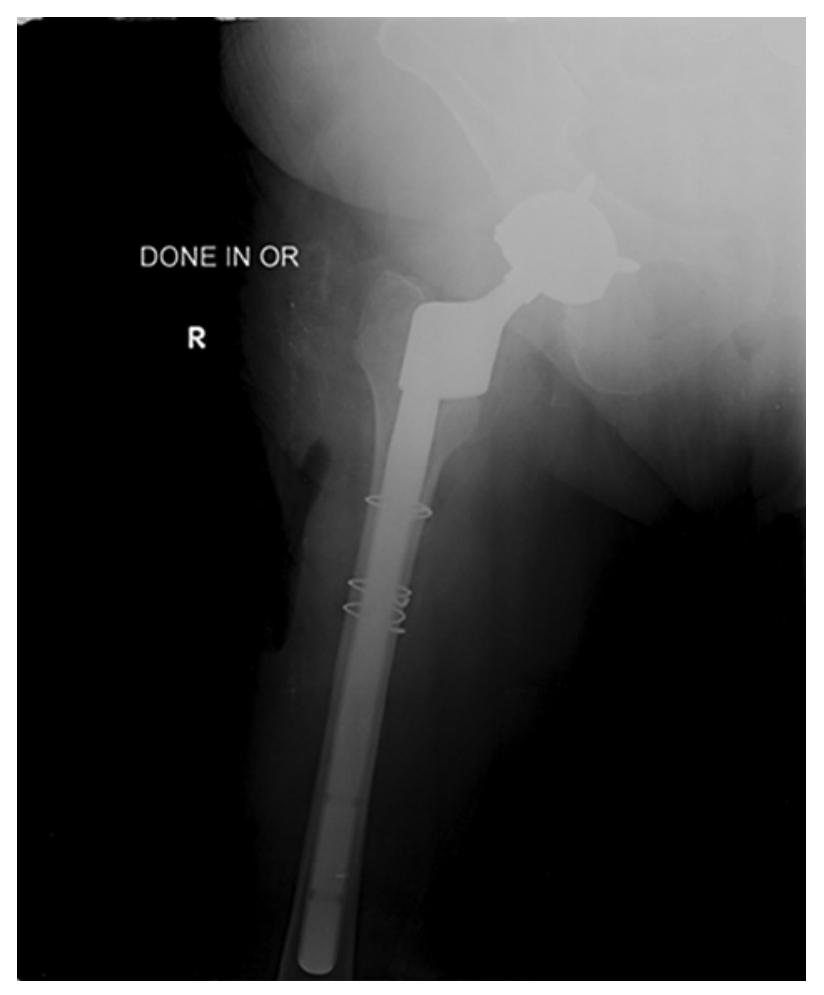

The patient was then started on Teicoplanin $400 \mathrm{mg}$ twice daily for broad-spectrum coverage. The cultures yielded E. coli ESBL producing strain that was sensitive to piperacillin/ tazobactam. The patient was therefore started on a course of piperacillin/tazobactam $4.5 \mathrm{~g} 3$ times daily. In the next few days, the patient began experiencing profuse diarrhea, and stool studies were positive for toxin A and B as well as for positive Clostridium difficile antigen. Metronidazole $500 \mathrm{mg} 3$ times daily is thus added to her regimen of treatment.

Ten days postoperatively, the patient began experiencing a fever and increase in inflammatory markers, as well as an increase in pus discharge from the surgical wound site. The patient was therefore scheduled for another lavage intervention. In addition to planning the debridement surgery, the patient was switched empirically to imipenem $500 \mathrm{mg} 4$ times 


\section{Case Reports in Orthopedic Research}
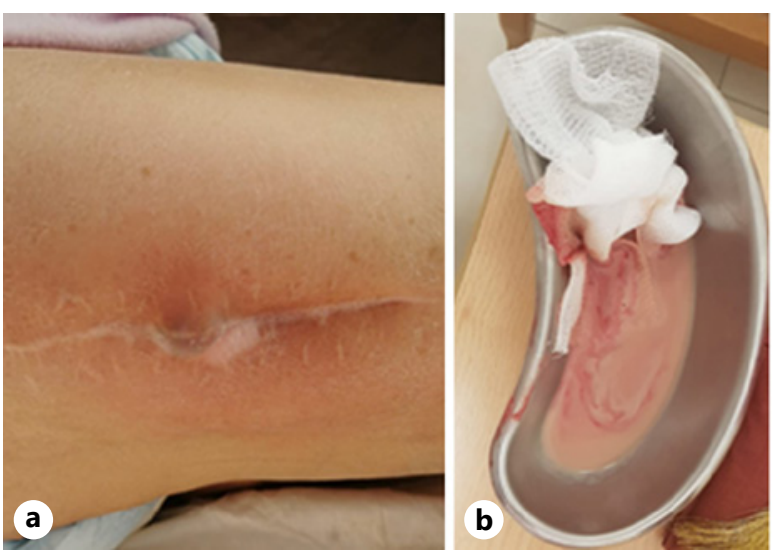

Fig. 6. Draining sinus with a subcutaneous collection (a); Pus drained during bedside lavage (b); wound healed and dry after serial lavage and dressings (c).

Fig. 7. Recent recurrence of superficial infected draining sinus.

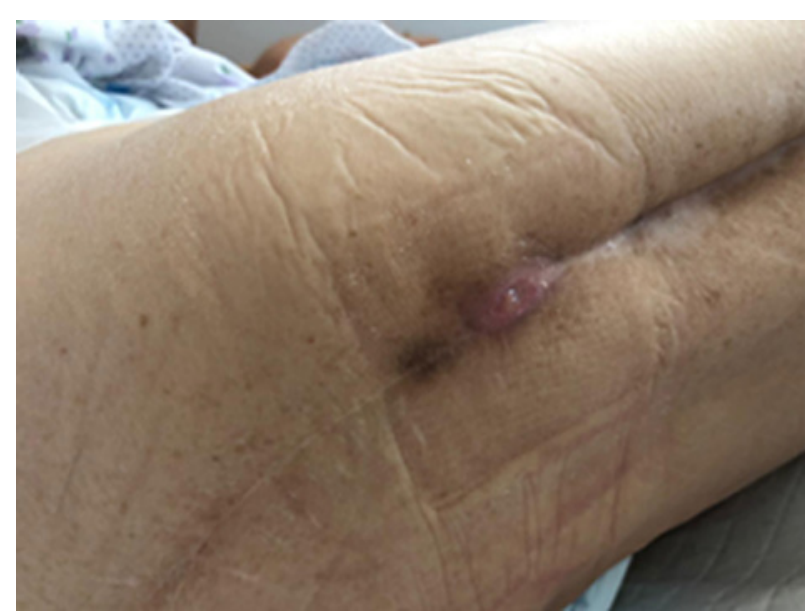

daily. During the operation, which also consisted of an intensive lavage with $12 \mathrm{~L}$ of normal saline and debridement, cultures were collected once more. Additionally, a vacuum dressing is applied. When the cultures came out showing ESBL producing E. coli species that is in fact intermediately resistant to piperacillin/tazobactam but sensitive to imipenem the patient was kept on her current imipenem and metronidazole antibiotic regimen during her 1 week of hospital stay. The patient is discharged to the nursing home, on a course of meropenem that lasted for 3 weeks, with a vacuum dressing that was regularly checked and changed.

Close monitoring of the patient's wound status was maintained by continuous communication with the nursing home. The wound healed well and the patient started on physiotherapy resuming ambulation with progressive weight-bearing achieving full weight-bearing at 6 weeks postop. Inflammatory markers are followed and showed to remain within a normal range.

Twenty-one months after discharge, while the patient is still in the nursing home the patient's surgical wound started oozing serous fluid (Fig. 6a, b) that was cultured and showed to be also E. coli of the ESBL Amp-C producing strain and that is sensitive to ertapenem. The patient is evaluated by infectious disease specialist and started on ertapenem treatment. Imaging showed no evidence of deep collection and the infection was considered to be superficial, without any other clinical symptoms such as fever and chills. The wound was treated 
with serial lavage, vacuum dressings, and ertapenem is resumed for 14 weeks until the infected site was clean, healed, and dry (Fig. 6c). The patient's scar remained in healed condition for 29 months, with an uneventful stay, described with functional ambulatory status and clean surgical site. After this period, she had a similar draining site (Fig. 7) from the surgical wound scar that is also treated with bedside lavage and was resolved in 4 weeks without affecting the patient's functional status nor causing systemic illness. The patient has been doing well since then, with no additional superficial infections noted, and she is still autonomous with a satisfactory functional status and walking distance.

\section{Discussion}

Infections around prosthetic joint replacements usually form biofilms, defined as an accumulation of micro-organisms that are embedded in a polysaccharide matrix and adherent to a solid surface [4]. The polymers that are produced by the bacteria forming the biofilm make it difficult for the immune system and even antibiotics to reach and eradicate these pathogens which can persist and lead to chronic infections. Presence of a biofilm can also reduce the inflammatory response and inflammatory markers, thus making even the diagnosis of a periprosthetic infection a possible challenge [4]. Note that $>50 \%$ of prosthetic joint infections are caused by gram-positive pathogens, specifically staphylococcus aureus and staphylococcus epidermidis. Other pathogens are common in scenarios of hematogenous spread such as gram-negative bacteria, streptococcus species, anaerobic bacteria, diphtheroids, and mycobacteria [5].

Various factors can contribute to treatment success when salvage of the prosthesis is opted for. The most important contributor is time from onset of symptoms. Targeting the treatment in the acute phase within 2 days of onset of symptoms is a very significant factor for success [6]. A BMI that is above 30 , and the presence of $>2$ comorbidities also increase the risk of recurrence and re-infection [7]. Staphylococcal organisms are significantly associated with higher failure rates [8]. In addition, a CRP level that is above $22 \mathrm{mg} / \mathrm{dL}$ is shown to predict a higher risk of failure in conservative treatment. Integrating vacuum dressings in management can enhance healing and infection control on various levels. In addition to drainage of collections and reduction of tissue bacterial counts [9], interstitial fluid alterations caused by vacuum dressing can modulate cellular wound-healing processes including growth factor expression and cellular mitotic activity. This can lead to an increase in transforming growth factor-B-1 and vascular endothelial growth factor expression, in addition to a 2 -fold increase in platelet-derived growth factor expression. It is also believed that the mechanical forces exerted on the extracellular matrix by negative pressure aid in decompensating small blood vessels and thus improve the wound's perfusion [10].

The success of retaining the prosthesis after debridement and lavage is highly variable in the literature [8]. Odum et al. [11] published a multicenter retrospective cohort study of 150 patients with a high failure rate up to $76 \%$. Another retrospective series of 138 joints by Koyonos et al. [6] also treated with debridement and irrigation shows a failure rate of $69 \%$ [8]. The same rate is also shown in the retrospective cohort study of Brandt et al. [12] on 30 patients with staphylococcus aureus periprosthetic infections after a 2-year follow-up.

On the other hand, other studies show high success rates after debridement and retaining the prosthesis, although those studies may be limited by a small sample sizes. In their prospective study between 1998 and 2004, Choong et al. [7] retained 11 out of 14 infected prostheses after debridement with 2 weeks of IV antibiotics, followed by 6 months of oral rifampicin. Meehan et al. [13] had an 89\% success rate after a 1-year follow-up in a retrospective study of 19 infected patients. A $90 \%$ success rate is reported in the study by Kelm et al. [9]

\section{Karger'}


on 28 patients where debridement is followed by vacuum dressing in 28 patients and the mobile components of the prosthesis are changed when possible [13]. Kim et al. [8] retrospectively studied 20 cases, treated with debridement and suction drains. All are recovered with no recurrence after follow-up to 55 months.

In our case, a 4-year follow-up is not only focused on the infection state, but also on evaluating the quality of life of the patient. Follow-up is based on revising notes from the nursing home describing the patient's daily activity, functional status, and ambulation. Over 4 years of follow-up, no recurrence is recorded except for 2 superficial infections at the surgical wound site that was treated by dressings, bedside lavage, and antibiotics in one of the 2 episodes with no further complications or effect on her ambulation status or function. As described in the study by Pavoni et al. [3], suppressing the infection, with salvage of the prosthesis and maintaining an acceptable functional status is a satisfactory result that is achieved in this selected case. This modality of treatment was chosen over a second revision surgery due many factors including patient age, comorbidities, and activity level. In the abovedescribed case, minimally invasive treatment modalities were opted in order to lower the risk of mortality of the patient, being a poor surgical candidate, preventing extensive surgical procedures causing blood loss and postoperative complications.

\section{Conclusion}

Periprosthetic hip joint infections can be a dreadful complication, especially in the elderly comorbid patients, rendering them poor surgical candidates. It is important to provide a welltailored management that fits each patient's profile. Emphasis should be done on close postoperative follow-up and patient compliance to prevent any further complications, especially when revision surgery puts the patient at high risk of morbidity.

\section{Statement of Ethics}

Approval and consent of the Ethics Committee have been received for the publication of this article. Written informed consent was obtained from the patient for publication of this case report and any accompanying images.

\section{Conflict of Interest Statement}

The authors declare that they have no competing interests.

\section{Funding Sources}

No funds were received in support of this study.

\section{Author Contributions}

M.D.: writing and editing the article, with editing of the images and radiographs. H.A.N.: writing and editing the article. E.S.: writing and editing the article. D.A.: writing and editing the article, with formatting and submission. G.L.: editing the text and data collection. J.H.: data 
collection and editing the article. A.N.: surgeon who supervised the case, writing, and finalizing the article.

\section{Availability of Data and Material}

The datasets used and/or analysed during the current study are available from the corresponding author on reasonable request.

\section{References}

1 Izakovicova P, Borens 0, Trampuz A. Periprosthetic joint infection: current concepts and outlook. EFORT Open Rev. 2019 Jul;4(7):482-94.

2 Qasim SN, Swann A, Ashford R. The DAIR (debridement, antibiotics and implant retention) procedure for infected total knee replacement: a literature review. SICOT-J. 2017;3:2.

3 Pavoni GL, Giannella M, Falcone M, Scorzolini L, Liberatore M, Carlesimo B, et al. Conservative medical therapy of prosthetic joint infections: retrospective analysis of an 8-year experience. Clin Microbiol Infect. 2004 Sep; 10(9):831-7.

4 Song Z, Borgwardt L, Høiby N, Wu H, Sørensen TS, Borgwardt A. Prosthesis infections after orthopedic joint replacement: the possible role of bacterial biofilms. Orthop Rev. 2013 Jun 7;5(2):65-71.

5 Tattevin P, Crémieux AC, Pottier P, Huten D, Carbon C. Prosthetic joint infection: when can prosthesis salvage be considered? Clin Infect Dis. 1999 Aug;29(2):292-5.

6 Koyonos L, Zmistowski B, Della Valle CJ, Parvizi J. Infection control rate of irrigation and débridement for periprosthetic joint infection. Clin Orthop. 2011 Nov;469(11):3043-8.

7 Choong PFM, Dowsey MM, Carr D, Daffy J, Stanley P. Risk factors associated with acute hip prosthetic joint infections and outcome of treatment with a rifampinbased regimen. Acta Orthop. 2007 Dec;78(6):755-65.

8 Kim JH, Chun SK, Yoon YC, Lakhotia D, Shon WY. Efficacy of debridement for early periprosthetic joint infection after hip arthroplasty. Hip Pelvis. 2014 Dec;26(4):227-34.

9 Kelm J, Schmitt E, Anagnostakos K. Vacuum-assisted closure in the treatment of early hip joint infections. Int J Med Sci. 2009 Sep 2;6(5):241-6.

10 Banwell PE, Musgrave M. Topical negative pressure therapy: mechanisms and indications. Int Wound J. 2004 Jun;1(2):95-106.

11 Odum SM, Fehring TK, Lombardi AV, Zmistowski BM, Brown NM, Luna JT, et al. Irrigation and debridement for periprosthetic infections: does the organism matter? J Arthroplasty. 2011 Sep;26(6 Suppl):114-8.

12 Brandt CM, Sistrunk WW, Duffy MC, Hanssen AD, Steckelberg JM, Ilstrup DM, et al. Staphylococcus aureus prosthetic joint infection treated with debridement and prosthesis retention. Clin Infect Dis. 1997 May;24(5): 914-9.

13 Meehan AM, Osmon DR, Duffy MCT, Hanssen AD, Keating MR. Outcome of penicillin-susceptible streptococcal prosthetic joint infection treated with debridement and retention of the prosthesis. Clin Infect Dis. $2003 \mathrm{Apr}$ $1 ; 36(7): 845-9$. 